\title{
Sweep circuit design for a picosecond streak camera
}

\author{
R J Bakert and B P Johnson‡ \\ †Department of Electrical Engineering, University of Idaho, Boise Engineering, \\ 1910 University Drive, TB201, Boise, Idaho 83725, USA \\ fDepartment of Electrical Engineering, University of Nevada, Reno, Reno, Nevada 89557, \\ USA
}

Received 6 September 1993, in final form 21 October 1993, accepted for publication 10 November 1993

\begin{abstract}
A review of the operation of a streak camera is presented. This is followed by a discussion of measurement techniques with time resolutions on the order 1 ps using the streak camera. A sweep circuit design is presented using power metal oxide semiconductor field effect transistors (MOSFETS). The sweep circuit generates two ramps

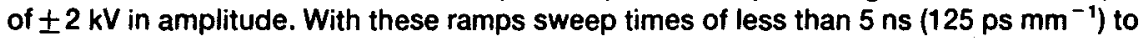
greater than $200 \mathrm{~ns}$ are achieved. The time-base variation is adjustable with a $D C$ voltage provided by a front-panel potentiometer (knob) similar to an oscilloscope.
\end{abstract}

\section{Introduction}

The streak camera is an instrument used to measure optical signals with time resolutions down to less than 1 ps [1]. The streak camera is composed of a streak tube, sweep electronics, microchannel plate image intensifier and a camera for reading the streaks (figure 1). Input light strikes the photocathode and generates electrons. These electrons are quickly accelerated towards the anode of the streak tube. The electron beam passes between the deflection plates and strikes the phosphor screen. Since the phosphor will stay illuminated for a time period that is long compared to the time the signal is present, a slow CCD camera or Polaroid film can be used to capture the signal.

The time history of the signal is obtained by knowing the sweep time, i.e. the time it takes the electron beam to traverse from the top of the phosphor screen to the bottom (figure 1). Often a comb generator is used as a

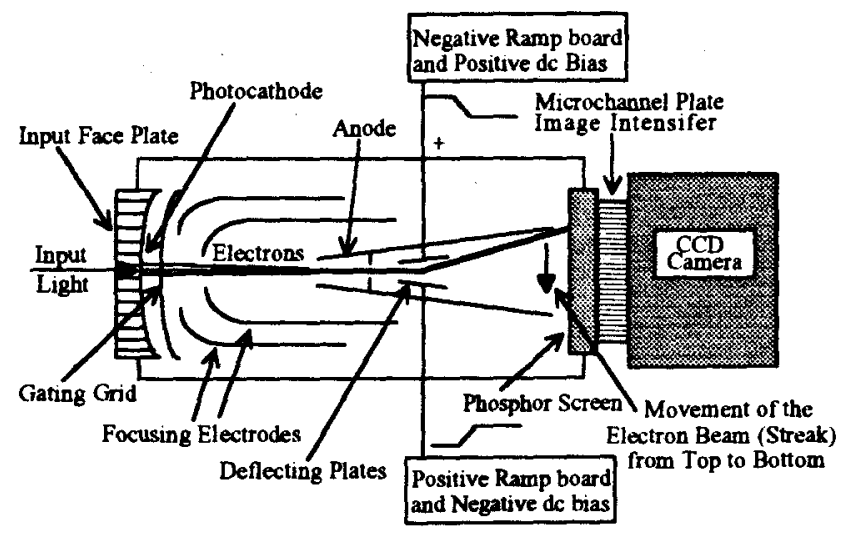

Figure 1. Diagram of the streak camera. time reference to measure sweep linearity and time. The comb generator produces a signal that is a series of impulses (or rectangular pulses for slower sweeps) separated by a known time. The signal generated looks like a comb, hence its name.

The amplitude (intensity) information is obtained by the brightness of the streak. For example, if a step function, $u(t)$ as shown in figure $2(a)$, is applied to the input when the sweep has travelled half way down the phosphor screen, a constant streak of light will appear from that point to the bottom of the screen. Figure $2(b)$ shows the streak obtained using a comb generator and figure $2(c)$ using a ramp.

For a $40 \mathrm{~mm}$ diameter streak tube, over 100 channels of data 'can be recorded simultaneously from large bandwidth signals. Applications of the streak camera might include (i) a real-time oscilloscope with a bandwidth greater than $50 \mathrm{GHz}$ using a Mach-Zehnder

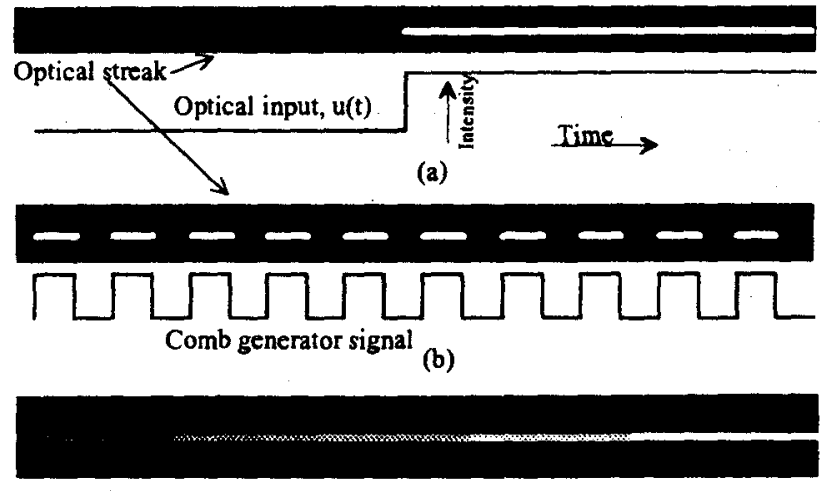

(c)

Figure 2. The optical streak recorded with (a) a stepped input, (b) a comb generator signal, (c) a ramped input. 
interferometer [2], and (ii) a non-invasive probe for ICs using the Pockels effect [3].

This paper is concerned with the sweep electronics (the ramp boards) portion of the streak camera. The deflection plates, shown in figure 1 , look like a capacitor of value $10 \mathrm{pF}$ (typically). Full deflection, i.e. the voltage required to move the electron beam from the top of the tube to the bottom, requires approximately $1.5 \mathrm{kV}$. Initially the electron beam is positioned off the screen, at the top, by the applied DC sweep plate voltages. After the sweep is completed, the electron beam is to be kept positioned off the screen at the bottom of the tube; this means that the ramps must be larger than $1.5 \mathrm{kV}$ required for full deflection.

To date, these high-voltage nanosecond ramps have been generated using avalanche transistors [4]. Avalanche transistors are difficult to use [5] because they lack (reproducibility, reliability and stability) and are nonlinear so external pulse shaping is needed. This increases the size and complexity of the sweep circuit and limits the range and number of different sweep times.

The design presented in this paper uses power MOSFETs that are operated linearly. A constant current is generated and applied to the deflection capacitance. This produces a linear ramping voltage between the deflection plates. The ramp rate is controlled by changing the magnitude of the current source (or the gate source voltage). Series operation of power MOSFErs [6] is used to generate the $\pm 2 \mathrm{kV}$ ramps needed.

\section{Design considerations}

The $10 \mathrm{pF}$ capacitance of the deflection plates is driven with a constant current. The positive ramp board sources current while the negative ramp board sinks current. In this way, two ramps of equal and opposite polarity can be generated.

Consider the simpler design with one of the deflection plates tied to ground and the other being modulated with a ramp. The electric field in the vertical direction does not change relative to the two-ramp case. However, the average potential (sum of the plate potentials divided by two) between the plates does change. In the tworamp case, the average potential remains constant for any given time. When one ramp is used, the potential is not constant. This causes problems with focusing, i.e. the streak seen on the phosphor becomes blurred thereby eliminating this mode as an option.

We can calculate the peak amount of current needed using the fastest sweep speed requirement. The amplitude of the ramps is picked to be $2000 \mathrm{~V}$. This gives an effective $4000 \mathrm{~V}$ ramp that is more than enough to overcome the DC biasing, which holds the electron beam off screen, deflects the beam across the screen, and holds the beam at the bottom after the event. If the fastest sweep time is $5 \mathrm{~ns}$, then the peak current is $10 \mathrm{pF}\left(4000 \mathrm{~V} / 5 \times 10^{-9} \mathrm{~s}\right)$ or $8 \mathrm{~A}$. Since only a portion of the ramp $(1.5 \mathrm{kV})$ will be used, the MOSFET current will be lower than this (typically in the range of $3 \mathrm{~A}$ for a
$5 \mathrm{~ns}$ sweep across $40 \mathrm{~mm}$ ). An alternate method of specifying the sweep rate is the time per $\mathrm{mm}$ of phosphor length. A $5 \mathrm{~ns}$ sweep across $40 \mathrm{~mm}$ corresponds to $125 \mathrm{ps} \mathrm{mm}^{-1}$. This is helpful when comparing relative sweep times of streak cameras.

\section{Circuit design}

Since we need two different ramp boards, it is desirable to have identical circuit designs for each board. One board would use positive supply voltages and n-channel devices and the other board would use p-channel devices with negative supply voltages. This means we must use complementary high-voltage MOSFETs.

The MOSFETs used in this design are the VN0345N2 n-channel and the VP0345N2 p-channel. A simplified schematic of the ramp circuits is shown in figure 3 . The bottom MOSFET in the stack controls the current through the other MOSFETs. If a constant gate source potential is applied between the gate and source of M1, then the

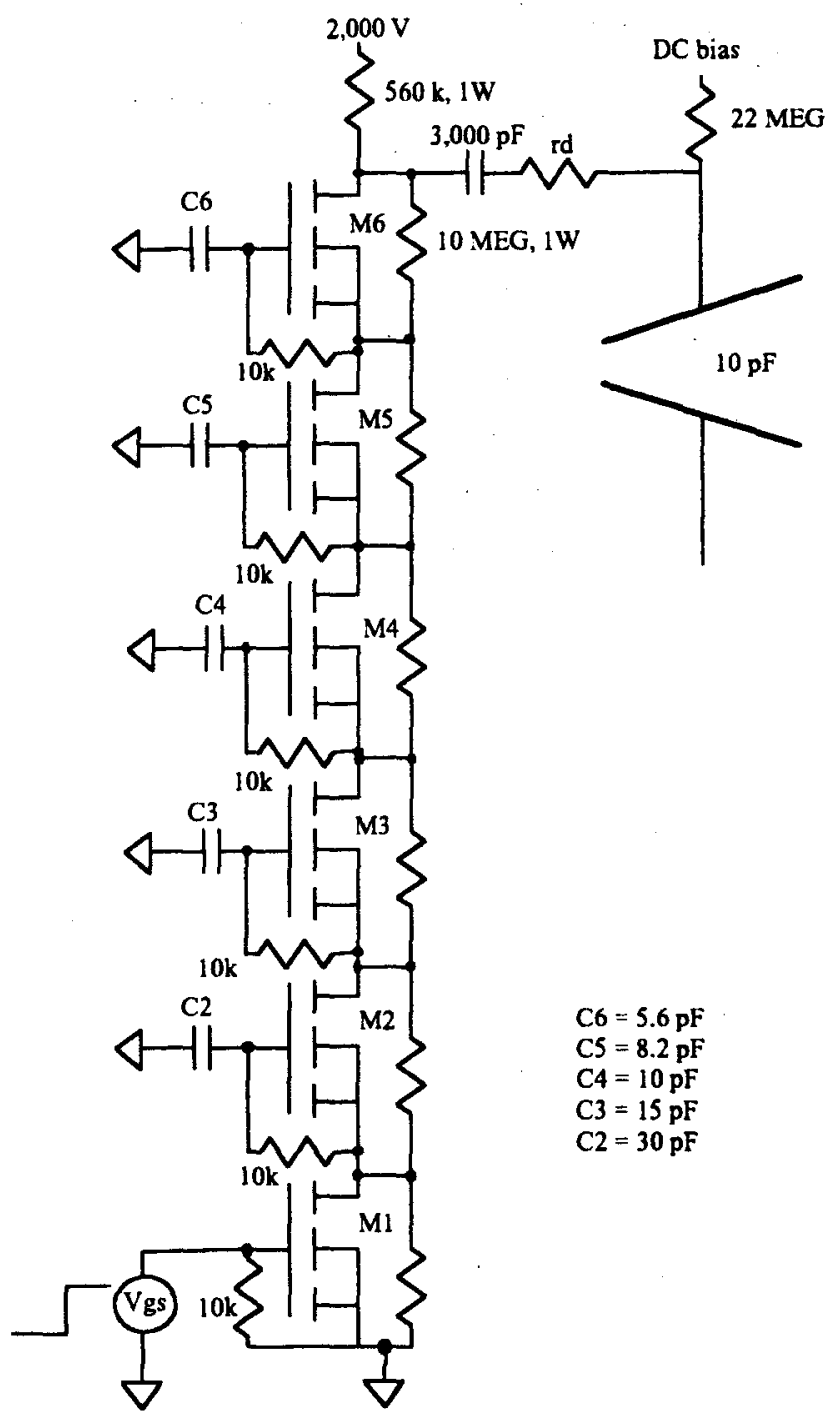

Figure 3. Simplified schematic of streak camera ramp board. The MOSFETS are V(N or P)0345N2. 
drain current is $g_{\mathrm{m}}\left(V_{\mathrm{gs}}-V_{\mathrm{th}}\right)$, providing the MOSFET stays in the saturation region. It is desirable to force all MOSFETs to turn on at the same rate to maximize overall linearity. If one of the MOSFETs turns on faster than the others, the ramp will have a discontinuity appearing as a change in slope.

The MOSFET gate-drain capacitance is $20 \mathrm{pF}$ and the gate-source capacitance is $550 \mathrm{pF}$. Following the method given by Baker and Johnson [6], the gate capacitors are: $C_{2}=30 \mathrm{pF}, C_{3}=15 \mathrm{pF}, C_{4}=10 \mathrm{pF}, C_{5}=$ $8.2 \mathrm{pF}$ and $C_{6}=5.6 \mathrm{pF}$ (figure 3 ). These capacitors form a capacitive voltage divider with the effective gate-source capacitance of each MOSFET. Their values are picked so that the maximum voltage across the gate-source junction is $10 \mathrm{~V}$.

Connecting the board to the deflection structure is accomplished using a wire. This jumper and the internal sweep plate connection is basically inductive. The resistor $r_{\mathrm{d}}$ is used to lower the $Q$ of the series $L C$ formed by the deflection capacitance and the interconnecting inductance. The value is picked so that the $2.2 R C$ risetime associated with the resistor deflection capacitance alone is small compared to the sweep time. In this case, $150 \Omega$ is used. This gives a risetime of $3.3 \mathrm{~ns}$ for the overall risetime. Faster sweeps can be achieved if this resistor is removed and M1 is driven with a $20 \mathrm{~V}$ step. This causes the MOSFET string to turn on rapidly. The limiting speed factor is the interconnecting inductance and the deflection capacitance.

The goal in this design was to replace the existing avalanche boards. The existing avalanche boards were settable to 5,10 , and 15 ns sweep speeds by replacing a wave-shaping module (low pass filter). Unless the camera was taken apart and the waveshaping module replaced, the camera was limited to one sweep speed. This makes it difficult to time complex systems. The new design allows us to change the sweep speed from 5 to $300 \mathrm{~ns}$ with a front panel knob (similar to an oscilloscope time base).

The knob controls the DC voltage source $V_{\mathrm{gs}}$ that, in turn, controls the ramp rate. The source is simply a DC source and a MOSFET switch. When the streak camera is triggered, the DC voltage is applied to the gate of M1.

Once the two circuit boards are in place, they must be aligned. The delay between the ramps is adjusted, as well as the slopes of the ramps. This is accomplished using two high-voltage oscilloscope probes (loading of approximately $1 \mathrm{pF}$ each). External delay is added or removed while comparing the mid-points on the ramps. The ramps are adjusted to have the same slope. One problem that exists is that although the slew rate of the ramps can be adjusted with the oscilloscope, the actual sweep time must be measured using the streak camera. This is caused by the DC voltages used for biasing the beam off of the screen, i.e. we do not know exactly when the beam will enter the phosphor screen.

\section{Results}

The most important attribute of the ramps is the linearity. Nonlinearities cause errors in amplitude as well as temporal information. Consider the streak shown in figure 2(c). Any nonlinearity in the ramp will cause the streak to become brighter in one place and darker in another affecting both the amplitude and time responses. Figure 4 shows the resulting ramps for the fastest sweep rate. The sweep time is approximately $4 \mathrm{~ns}$ and the linearity is better than $2 \%$. This sweep time was limited by the speed of the $\mathrm{p}$-channel board. The n-channel board could generate ramps at least two times faster than the p-channel MOSFET board.

The linearity data shown in figure 4 were determined using the $3 \mathrm{GHz}$ comb shown in figure 5 . The comb is used to determine the actual sweep speed. If the ramps are perfectly linear, the time difference between adjacent

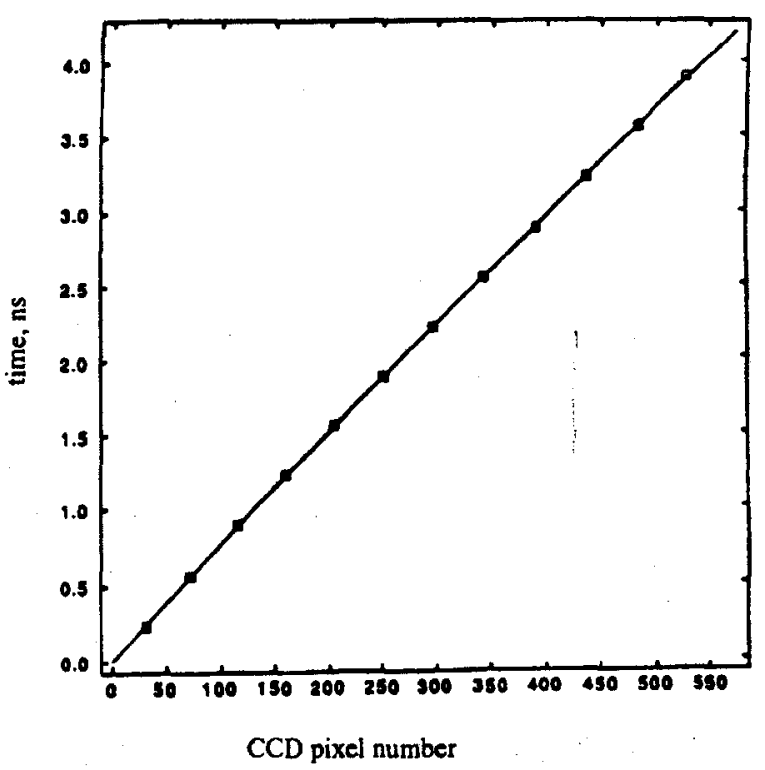

Figure 4. Shows sweep time of approximately 4 ns. $\square--\square$, time peaks; —, order 2 fit.

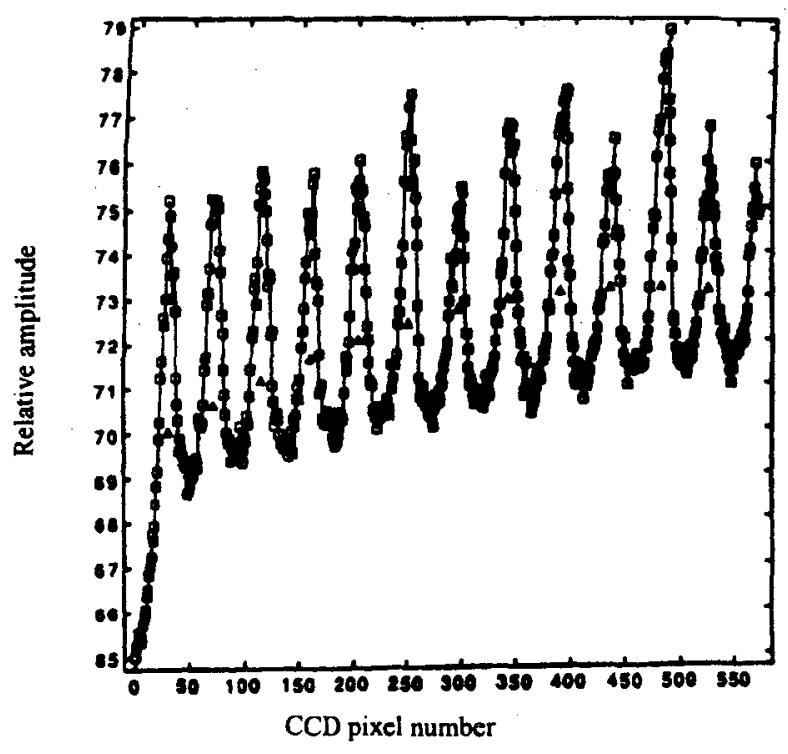

Figure 5. Shape of the $3 \mathrm{GHz}$ comb used for calculating the sweep time. 
peaks in figure 5 will be equal. The differences in the times is used to determine the nonlinearity.

For the 4 ns sweep shown in figure 4 , the MOSFETs are well within the saturation region. As the sweep time is increased, and the constant current is smaller, the MOSFETs come close to the cut-off region. When this occurs, the drain current no longer follows the relationship $g_{\mathrm{m}}\left(V_{\mathrm{gs}}-V_{\mathrm{th}}\right)$ but becomes nonlinear. This causes nonlinearities in the sweep. Figure 6 shows this effect. The sweep here is approximately $160 \mathrm{~ns}$ and the nonlinearity is $5 \%$. As the sweep is lengthened to $300 \mathrm{~ns}$, the nonlinearity increases to $10 \%$.

One solution to this problem is to place a capacitor in parallel with the deflection capacitance as the sweeps are lengthened. This can be accomplished with a relay or switch that detects when the capacitor is needed. For our applications, the longer sweeps were mainly used as a coarse setting to determine where the signal is in time. The linearity of the fast sweeps is the most important parameter for good data in our measurements.

\section{Conclusion and discussion}

Although the design presented here is a significant improvement over previously used designs, there is still need for improvement. It is desirable to have a faster sweep time, for example $1 \mathrm{~ns}$. This could be accomplished using the 'brute force' method by increasing the amplitude of the ramps and using the inductance between the ramp board and the deflection capacitance to peak the voltage across the deflection structure. However, as the amplitude increases beyond $3 \mathrm{kV}$, components become more difficult to find, more inductive, and more safety precautions must be taken.

Future work will involve designs for faster ramps, eliminating the difference in time delay between ramps and having automatic slope adjustment between the p- and n-channel MosfeT boards. Our design goal is to attain a sweep circuit variable from $1 \mathrm{~ns}\left(25 \mathrm{ps} \mathrm{mm}^{-1}\right)$ to $300 \mathrm{~ns}$ with little alignment required.

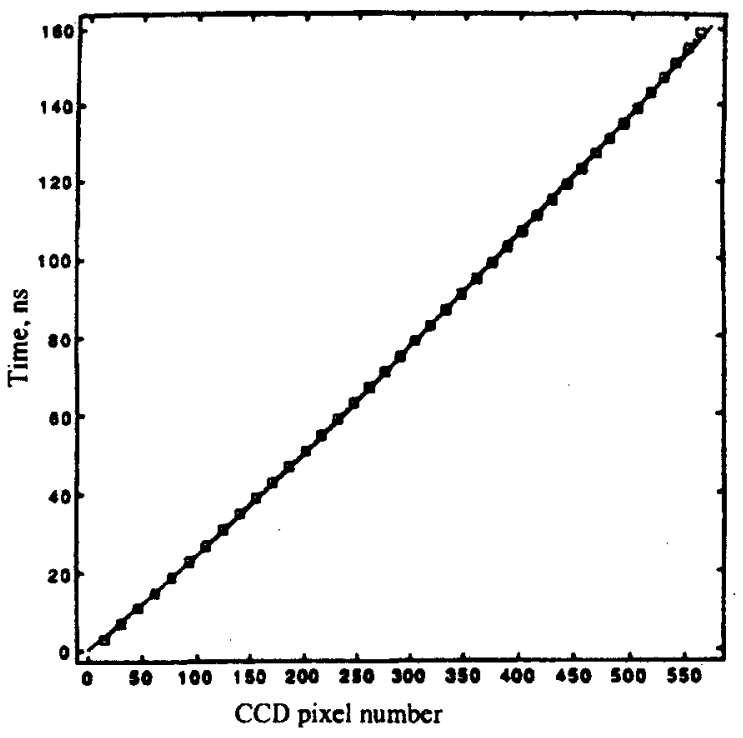

Figure 6. A longer sweep showing the effect of coming close to the cut-off region. $\square---\square$, time peaks: - order 2 fit.

\section{References}

[1] Tsuchiya Y. 1983 Picosecond streak camera and its applications (comprehensive information on streak cameras) Picosecond 14

[2] Williamson S and Mourou G A 1985 Picosecond electro-electron optic oscilloscope Picosecond Electronics and Optoelectronics, Springer Series in Electrophysics Vol 21 ed DM Mourou et al (Berlin: Springer) pp 58-61

[3] Leonberger F. J, Lee C H, Capasso F and Morkoc $\mathrm{H}$ (ed) 1987 Picosecond Electronics and Optoelectronics II, part 1, Springer Series in Electronics and Photonics Vol $24 \mathrm{pp}$ 4-71

[4] Thomas S W. Griffith R L and McDonald W R 1986 Improvements in avalanche-transistor sweep circuity for electro-optic streak cameras Opt. Eng. 25 465-70

[5] Bakar R J 1991 High voltage pulse generation using current mode second breakdown in a bipolar junction transistor Rev. Sci. Instrum 62 1031-6

[6] Baker R J and Johnson B P 1992 Stacking power MOSFET for use in high speed instrumentation Rev. Sci. Instrum 63 5799-801 